\title{
Variación anatómica en la formación de los nervios mediano y musculocutáneo
}

\author{
(iD) Roberto Mejías Stuven ${ }^{1}$, Juan Carlos Coronado Lopez ${ }^{2}$, Eugenia Carvallo Semler ${ }^{3}$
}

\section{RESUMEN:}

C ntre los nervios mediano y musculocutáneo se establecen comunicaciones que determinan variaciones en la formación de ambas estructuras Enerviosas. Patologías o traumas de las regione axilar o braquial que deriven en compromiso de éstos elementos nerviosos, hace fundamental el conocimiento de ellos para la práctica clínica o quirúrgica. En este trabajo presentamos un caso de anomalía en la formación de los nervios mediano y musculocutáneo, que determina una variable en la comunicación entre ambos nervios terminales.

PALABRAS CLAVE: Nervio mediano; Nervio musculocutáneo; Comunicación nerviosa.

\section{Anatomical variation in the formation of the median and musculocutaneous nerves SUMMARY:}

\begin{abstract}
Retween the median and musculocutaneous nerves are established communications that determine variations in the formation of both Bneural structures. The knowledge of them is of fundamental importance in clinical and surgical practice, in the presence of diseases or trauma of the axillary or brachial arteries and whose intervention resulting in compromise of these neural elements. We present a case of abnormality in the formation of the median and musculocutaneous nerves, which determined the establishment a variable communication between both terminals trunks.
\end{abstract}

KEYWORDS: Median nerve; Musculocutaneous nerve; Nerve communication.

\section{INTRODUCCIÓN}

T as variaciones nerviosas del plexo braquial presentan Lun correlato clínico y quirúrgico de importancia en la práctica médica. Los nervios mediano y musculocutáneo son dos ramas terminales del plexo braquial.

EEl plexo braquial deriva de las raíces ventrales de $\mathrm{C} 5$, C6, C7, C8 y T1, pudiendo participar en forma variable C4 y $\mathrm{T} 2$. El tronco primario superior se forma a partir de $\mathrm{C} 5$ y C6, el tronco medio se forma a partir de C7 y el tronco primario inferior es formado por $\mathrm{C} 8 \mathrm{y} \mathrm{T} 1$. Cada tronco primario se divide en ventral y dorsal, constituyendo los troncos secundarios. Las divisiones dorsales de los tres troncos $(\mathrm{C} 5, \mathrm{C} 6, \mathrm{C} 7, \mathrm{C} 8, \mathrm{~T} 1)$ forman el fascículo dorsal. Las divisiones ventrales de los troncos superior y medio (C5, C6, C7) forman el fascículo lateral, mientras que la división ventral del tronco inferior $(\mathrm{C} 8, \mathrm{~T} 1)$ forman el fascículo medial. El nervio mediano se encuentra formado por dos raíces, originadas de los fascículos lateral y medial del plexo braquial.

Estas rodean la tercera parte de la arteria axilar, ventral o lateralmente a ella, y sus fibras proceden de las raíces ventrales de C6-C7 para el fascículo lateral y C8-T1 para el fascículo medial. Por otro lado, el nervio musculocutáneo se origina del fascículo lateral del plexo braquial, ubicándose lateral a la arteria axilar, con fibras procedentes de las raíces nerviosas ventrales C5 y C6 (1-5).

Las comunicaciones entre los nervios musculocutáneo y mediano, las anomalías en su formación y trayecto, han sido registradas con anterioridad (1,6-11). El desconocimiento de la probable presencia de estas anormalidades nerviosas, las convierte en estructuras propensas a sufrir lesiones durante el curso de la disección y la cirugía. Por eso consideramos que el conocimiento de las variaciones de los nervios de la extremidad superior es de fundamental importancia en los traumatismos y patologías de la región, ya que, del oportuno diagnóstico y tratamiento de los mismos, en la clínica y la cirugía, depende el mantenimiento de la correcta funcionalidad del miembro superior.

En este trabajo presentaremos una variación en la comunicación nerviosa establecida entre los nervios mediano y musculocutáneo, y que determinará una formación y trayecto de estos troncos nerviosos que difiere de la descripción clásica.

\section{MATERIAL Y MÉTODO}

Se realizó la disección de rutina de las regiones axilar y braquial izquierdas de un cadáver de sexo masculino, caucásico, de edad indefinida, conservado en formalina al $2 \%$ y ácido acético al $0,5 \%$, en el laboratorio de anatomía de la Universidad Mayor,Temuco.

\section{RESULTADOS}

Macroscópicamente, se observó que ambas raíces que originan el nervio mediano, inmediatamente a su emergencia se unían, ubicándose en superior y luego lateralmente a la arteria axilar. Ambos nervios cruzaban la axila e ingresaban al brazo en relación con el músculo axilopectoral, o músculo axilar (de Langer), variación muscular de la axila que, siguiendo su disposición más frecuente, se origina del músculo dorsal ancho, extendiéndose por delante del paquete vasculonervioso de la axila, para insertarse distalmente en el músculo pectoral mayor, a nivel de su inserción humeral. El nervio musculocutáneo, junto al nervio mediano, se separaban del músculo y continuaban su trayecto normal, perforando, el

\footnotetext{
1. Temuco, Chile. Doctorado en Ciencias Morfológicas, UFRO.

2. Universidad Católica, Temuco, Chile. Magíster Ciencias de la 3 Salud mención Morfología, UFRO.

3. Universidad Mayor, Temuco, Chile. Magíster Ciencias de la Salud mención Morfología, UFRO

Citar como :Mejías Stuven R, Coronado Lopez JC, Carvallo Semler E. Variación anatómica en la formación de los nervios mediano y musculocutáneo. Rev Peruana de Morfologia. 2020; 1(1): 4-5

Recibido: 26/07/2020; Aceptado: 12/10/2020
}

Autor corresponsal: Roberto Mejías Stuven. ORCID: https://orcid.org/0000-0002-7156-4319 email: roberto.mejias@gmail.com; 
nervio musculocutáneo al músculo coracobraquial. Casi la totalidad de las fibras del fascículo lateral del nervio mediano se adosaban al nervio musculocutáneo y seguían con él hasta el brazo (Fig. 1).

El nervio musculocutáneo daba origen a las ramas nerviosas destinadas a los músculos coracobraquial, bíceps braquial, y braquial, para quedar reducido finalmente a un pequeño nervio. En la parte distal del tercio medio del brazo, el nervio musculocutáneo emitió una delgada

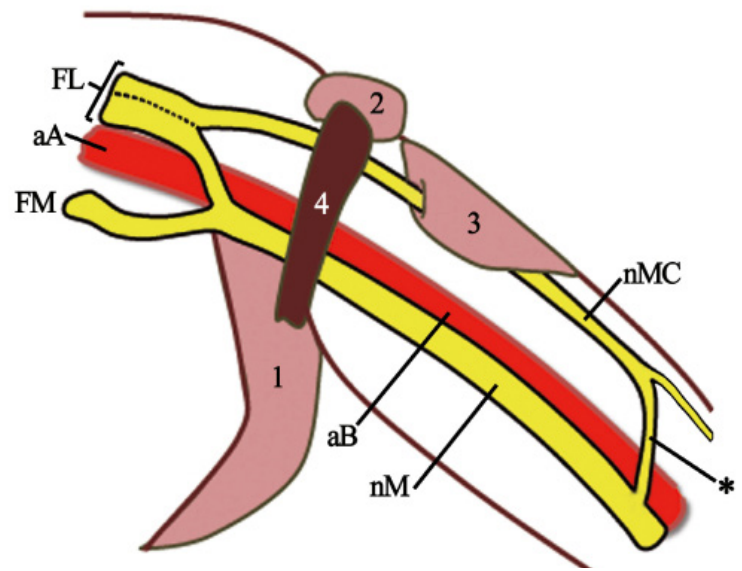

Fig. 1. Representación esquemática del caso presentado en este artículo. FL, fascículo lateral del plexo braquial; FM, fascículo medial del plexo braquial; $\mathbf{a A}$, arteria axilar; $\mathbf{a B}$, arteria braquial; $\mathbf{n M}$, nervio mediano; nMC, nervio musculocutáneo; 1, músculo dorsal ancho; 2, músculo pectoral mayor; 3, músculo coracobraquial; 4, músculo axilar (de Langer).* Comunicación nerviosa entre el nMC y el nM en la parte distal del tercio medio del brazo.

rama de comunicación destinada al nervio mediano, que consistía en fibras de la raíz lateral pertenecientes al nervio mediano, que acompañaban al musculocutáneo durante todo su trayecto en el brazo (Figs. 2,3 y 4).

\section{DISCUSIÓN}

La anastomosis entre los nervios mediano y musculocutáneo es una situación anatómica que se observa con frecuencia, y sus distintas maneras de comunicación han sido reportadas en la literatura $(1,6-11)$.

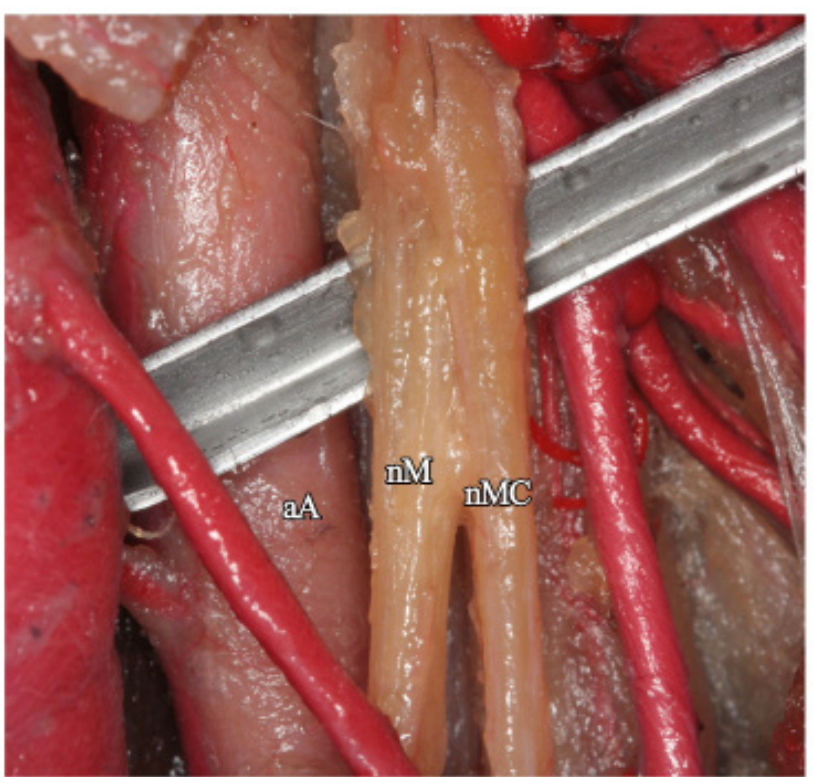

Fig. 2. Región axilar izquierda: formación del nervio mediano (nM) y el nervio musculocutáneo (nMC) lateral a la arteria axilar (aA).

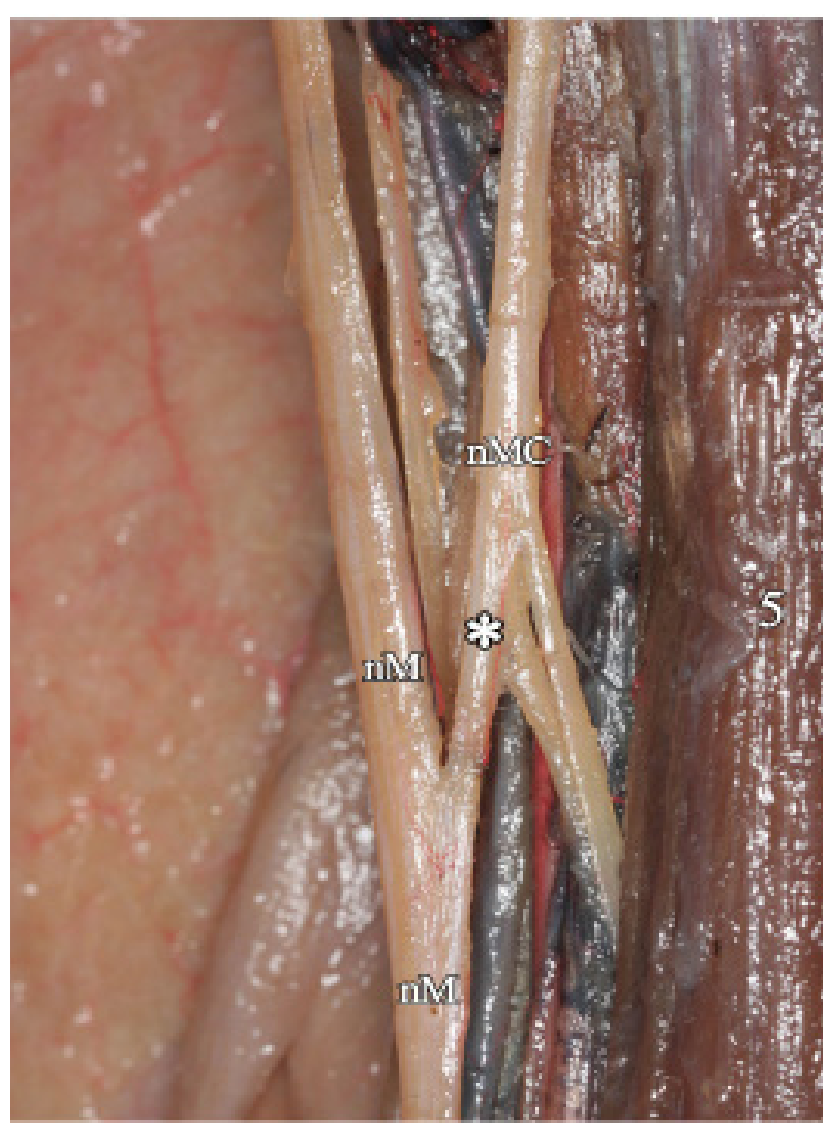

Fig. 3. Región braquial izquierda: se observa la comunicación nerviosa (*) entre el nervio mediano (nM) y el nervio musculocutáneo (nMC) a nivel de la parte distal del tercio medio del brazo, medial al músculo bíceps braquial.

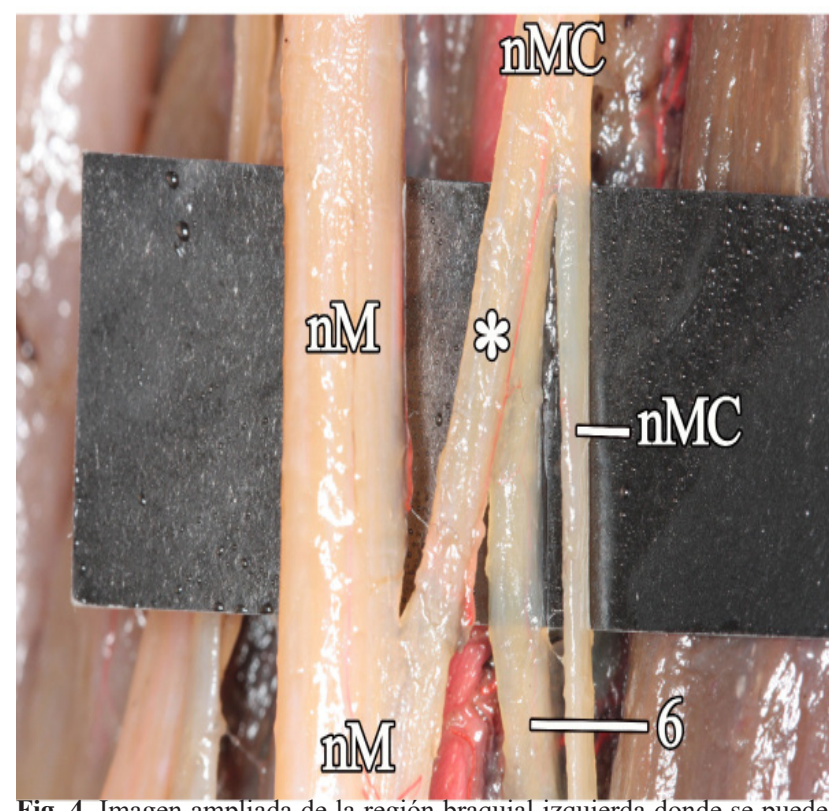

Fig. 4. Imagen ampliada de la región braquial izquierda donde se puede observar la comunicación nerviosa ( *) entre los nervios mediano (nM) y musculocutáneo (nMC). Se ve la continuación del nMC como una delgada rama nerviosa, y también la emisión de un ramos nervioso para el músculo bíceps braquial (6).

Las variaciones de los troncos de formación del plexo braquial fueron descriptas por Kerr (1918) en 11 de 175 casos (12). Por su parte, Walsh (1877) describió anomalías en 2 de 350 plexos braquiales disecados (13).

Bergman et. al. (2006) describen los estudios de Hartmann, quien en 1888 realizó una descripción de las 
variaciones en la anastomosis entre los nervios mediano y musculocutáneo, en los cuales siempre se encontraba la raíz lateral a nivel axilar, de la cual se originaban el musculocutáneo y el mediano, para luego establecerse la variación en la anastomosis desarrollada a nivel del brazo (14).

Kaus y Wotowicz (1995) describieron la comunicación del nervio musculocutáneo hacia el nervio mediano en ambos lados de un cadáver (15).

Li Minor (1992) desarrolla una clasificación describiendo cinco tipos de comunicaciones (16). En el tipo I, no hay comunicación entre el mediano y el musculocutáneo; en el tipo II, parte de las fibras de la raíz lateral correspondientes al mediano se unen al nervio musculocutáneo y lo acompañan en su recorrido para luego volver al mediano en la mitad del brazo; en el tipo III, todas las fibras del fascículo lateral del plexo braquial conforman el musculocutáneo y las correspondientes al mediano lo alcanzan distalmente para formar su raíz lateral. En el tipo IV, las fibras del fascículo lateral correspondientes al musculocutáneo se unen a la raíz lateral del mediano, conformando este nervio y después de una cierta distancia surge el nervio musculocutáneo. En el tipo V, el musculocutáneo está ausente y las fibras del fascículo lateral que le corresponden pasan al mediano a través de su raíz lateral, el cual posteriormente proporciona los ramos nerviosos para los músculos inervados por el musculocutáneo. Adachi (1918) reportó una comunicación muy delgada entre los nervios mediano y musculocutáneo en el antebrazo, ubicada por detrás de la arteria radial a nivel del codo (17). Lang y Spinner (1970) describieron una fusión completa entre el nervio mediano y musculocutáneo. Con respecto a esta última disposición, Watanabe et al. (1985) encontraron dos casos, en un total de 140 miembros superiores investigados $(1,4 \%)(18)$.

Con respecto a nuestro caso, se enmarcaría dentro del tipo II de Li Minor, en el cual el nervio mediano se encuentra disminuido en su conformación nerviosa, ya que parte de sus fibras del fascículo lateral del plexo braquial continúan su recorrido junto al nervio musculocutáneo, presentándose una raíz lateral adelgazada, y conformándose un nervio musculocutáneo de mayor grosor. Este, a nivel de la mitad del brazo, devuelve al nervio mediano sus fibras nerviosas estableciendo una anastomosis nerviosa, continuando el nervio musculocutáneo, como un elemento nervioso de menor magnitud.

Es posible que esta variación anatómica de los nervios mediano y musculocutáneo, que no es infrecuente, se deba a que fibras del fascículo lateral del nervio mediano se desprenda a nivel axilar y se unan al nervio musculocutáneo. Esto último explicaría la pequeña raíz lateral del nervio mediano que, en este caso, corre junto al nervio musculocutáneo. La interpretación del curso atípico de estos ramos nerviosos del fascículo lateral del plexo braquial se relaciona con el estudio del desarrollo y evolución de la inervación y musculatura del miembro superior. Alteraciones en la expresión de las células mesenquimales y de crecimiento neuronal determinan modificaciones no solo en la formación de los nervios, sino también, en su recorrido e inervación muscular (19).

Las anomalías de comunicación de los nervios del miembro superior suelen ir acompañadas de variaciones vasculares. Hollinshead (1982) describe este tipo de variaciones en la formación de los cordones del plexo braquial y su diferentes disposiciones en relación a variaciones de las arterias subclavia y axilar (20).

Es importante recordar, por lo tanto, la anatomía del fascículo lateral del plexo braquial, cuyas variaciones van a determinar la conformación, normal o variable, de los nervios mediano y musculocutáneo y la posibilidad de desarrollo de comunicaciones entre la axila y el codo. Esta anatomía variable deberá conocerse para realizar intervenciones quirúrgicas en la región del hombro, bloqueos nerviosos, artroscopías y cirugía reconstructiva del hombro $(4,21)$. Será de gran valor el reconocimiento adecuado de las estructuras nerviosas y sus diferentes formas de disposición, para poder realizar un tratamiento quirúrgico adecuado y seguro.

\section{Referencias bibliograficas}

1. Linell EA. The Distribution of Nerves in the Upper Limb, with reference to Variabilities and their Clinical Significance. J Anat. 1921;55(2):79-112.

2. Testut JL, Latarjet A. Anatomía Humana. 9 ed. Barcelona: Salvat Editores S.A; 1980.

3. Venieratos D, Anagnostopoulou S. Classification of communications between the musculocutaneous and median nerves. Clin Anat. 1998;11(5):327-31.

4. Bollini CA. Revisión anatomica del plexo braquial. Rev Arg Anest. 2004;62(6):386-98.

5. Rouviére H, Delmas V, Delmas A. Anatomía humana descriptiva, topográfica y funcional. 11․ Barcelona: Ed Masson; 2005.

6. Uzun A, Seelig LL. A variation in the formation of the median nerve: communicating branch between the musculocutaneous and median nerves in man. Folia Morphol. 2001;60(2):99101

7. Choi D, Rodríguez-Niedenführ M, Vázquez T, Parkin I, Sañudo JR. Patterns of connections between the musculocutaneous and median nerves in the axilla and arm. Clin Anat. 2002;15(1):11-7.

8. Saeed M, Rufai AA. Median and musculocutaneous nerves: Variant formation and distribution. Clin Anat. 2003;16(5):453-7.
9. Beheiry EE. Anatomical variations of the median nerve distribution and communication in the arm. Folia Morphol. 2004;63(3):313-8.

10. Loukas M, Aqueelah H. Musculocutaneous and median nerve connections within, proximal and distal to the coracobrachialis muscle. Folia Morphol. 2005;64(2):101-8.

11. Marcante Carlotto JR, Ambros LE, Dal Vesco JA, Reichert PR. Origen y Trayecto Anómalos del Nervio Musculocutáneo Anomalus Origin and Traject of Musculocutaneous Nerve. Vol. 27, Int. J. Morphol. 2009.

12. Kerr AT. The brachial plexus of nerves in man, the variations in its formation and branches. Am J Anat. 1918;23(2):285-395.

13. Walsh J. The anatomy of brachial plexus. Am J M Sci. 1877;74(1):387-428.

14. Bergman R., Afifi AK, Miyauchi R. Variety of Anastomoses between the Musculotaneous and Median Nerves. Illustrated Encyclopedia of Human Anatomic Variation: Opus III: Nervous System: Plexuses: Musculocutaneous Nerve. 2006. Disponible en: http://www. anatomyatlases.org/AnatomicVariants/ NervousSystem/Images/hartmann.shtml

15. Kaus M, Wotowicz Z. Communicating branch between the musculocutaneous and median nerves in human. Folia Morphol. 1995;54(1):273-7.

16. Li Minor JM. A rare variation of the median and musculocutaneous nerves in man. J Morphol Sci. 2011;28(4):246-9.

17. Adachi B. Das Arterien system der Japaner. Univ. Kyoto; 1928. 201-2 p.

18. Watanabe M, Takatsuji K, Sakamoto $\mathrm{N}$, Morita Y, Ito $\mathrm{H}$. Two cases of fusion of the musculocutaneous and median nerves. Acta Anat Nippon. 1985;60(1):1-7.

19. Abhaya A, Khanna J, Prakash R. Variation of the lateral cord of brachial plexus piercing coracobrachialis muscle. J Anat Soc India. 2003;52(2):168-70.

20. Hollinshead WH. Anatomy for surgeons. 2 ed. Harper and Row Publishers, editor. Vol. 3, British Journal of Surgery. New York: Hoeber Medical Division; 1982.

21. Ortega Romero A, de Isasa D, Del Olmo Rodríguez C, Maroto Ramos E, Rouco Gil R. Portable ultrasound devices in regional anesthesia: the brachial plexus block. Rev Esp Anestesiol Reanim. 2008 1;55(5):294-303. 